\title{
HAUSDORFF DIMENSION OF DIVERGENT TEICHMÜLLER GEODESICS
}

\author{
HOWARD MASUR
}

\begin{abstract}
Let $g>1$ be given and let $k=\left(k_{1}, \ldots, k_{n}\right)$ be an $n$-tuple of positive integers whose sum is $4 g-4$. Denote by $Q_{k}$ the set of all holomorphic quadratic differentials on compact Riemann surfaces of genus $g$ whose zeros have orders $k_{1}, \ldots, k_{n} . Q_{k}$ is called a stratum inside the cotangent space of all holomorphic quadratic differentials over the Teichmüller space of genus $g$. Let $Q_{k} / \operatorname{Mod}(g)$ be the moduli space where $\operatorname{Mod}(g)$ is the mapping class group. Each $q \in Q_{k}$ defines a Teichmüller geodesic.

Theorem. There exists $\delta>0$ so that for almost all $q \in Q_{k}$, the set of $\theta$, such that the geodesic defined by $e^{i \theta} q$ eventually leaves every compact set in $Q_{k} / \operatorname{Mod}(g)$, has Hausdorff dimension $\delta$.
\end{abstract}

\section{INTRODUCTION}

Suppose we consider a hyperbolic line $L$ in the upper half plane $H$ with endpoint $\zeta$ on $\mathbf{R}$. The fundamental domains for the $S L(2, Z)$ action on $H$ tile $H$. $L$ goes through a sequence of the domains. Each can be mapped to the standard fundamental domain. We can ask whether $L$ diverges to $\infty$ in the sense that it eventually leaves every compact set in the fundamental domain. It is not hard to establish that $L$ diverges if and only if $\zeta$ is rational. In this case the sequence of fundamental domains is finite and under the last translation $L$ is mapped to a vertical line to $\infty$.

Now $H$ is the Teichmüller space of a torus, $H / S L(2, Z)$ is the moduli space, and the hyperbolic lines are Teichmüller geodesics. The condition that $\zeta$ is rational means that the Teichmüller maps defining the geodesic are pinching a simple closed curve. The set of tangent vectors through each point of $H$ defining a divergent geodesic is countable; thus the subset of the unit tangent bundle is a countable union of sets of two dimensions and so has Hausdorff dimension one less than the dimension of the total space.

Received by the editors April 22, 1988 and in revised form March 3, 1989.

1980 Mathematics Subject Classification (1985 Revision). Primary 32G15, 30F30; Secondary $58 \mathrm{~F} 17$.

The author was supported in part by National Science Foundation grant DMS 8601977. 
In this paper we investigate the phenomenon of divergence of Teichmüller geodesics in moduli spaces of quadratic differentials on surfaces of genus $g>1$.

A Teichmüller geodesic $L$ through a Riemann surface $X$ is defined by a quadratic differential $q$ on $X$. For each real $t$ one defines a Teichmüller map to an image surface $X_{t}$ on $L$ by contracting by $e^{t / 2}$ along the vertical trajectories of $q$, and expanding by $e^{t / 2}$ along the horizontal trajectories. The image or terminal quadratic differential $q_{t}$ on $X_{t}$ has the same orders of zeros as $q$. We say the geodesic diverges if for any $\varepsilon>0$, for all sufficiently large $t$, $q_{t}$ has a saddle connection (geodesic joining two zeros) of length $\leq \varepsilon$. In the special case where $q$ has only one zero, this means there is for sufficiently large $t$ a short simple closed curve. One way to achieve divergence as in the torus case is to "pinch" along a saddle connection. If $q$ has a saddle connection which is a vertical trajectory, then the length of the saddle connection goes to zero along the geodesic. As in the case of the torus the set of $q$ on each Riemann surface $X$ defining such a pinching geodesic is small, i.e., a countable union of sets of positive codimension.

A new phenomenon occurs however in $g>1$. A geodesic may diverge without a fixed saddle connection becoming short. Rather there is an infinite set of distinct saddle connections such that for any sufficiently large time at least one of the connections is arbitrarily short but any fixed connection eventually becomes long. At certain times two or more connections are short. We call such divergent geodesics spiralling. Our theorem says the Hausdorff codimension of the quadratic differentials that define spiralling geodesics in the total space of all quadratic differentials is less than one when $g>1$.

That such spiralling geodesics might exist is suggested by the fact that the moduli space, or stratum, of quadratic differentials all with the same orders of zeros is not compact. It can be compactified by adding a finite number of lower dimensional strata consisting of quadratic differentials on surfaces of lower genus or quadratic differentials with higher order zeros on surfaces of the same genus. Then a geodesic might successively approach one boundary strata after another. In the special case of a quadratic differential with a single zero, the boundary strata are quadratic differentials on surfaces of lower genus. Thus in the context of moduli spaces of Riemann surfaces, the underlying Riemann surfaces $X_{t}$ on the geodesic are successively approaching different moduli spaces of surfaces of lower genus. When $g=1$ this phenomenon is not possible since the boundary of moduli space is a point. The first example of this spiralling was constructed by Kerckhoff [K].

In order to state the theorem precisely we need the following notation. Let $M$ be a compact $C^{\infty}$ surface of genus $g>1$. Let $\Sigma=\left(x_{1}, \ldots, x_{n}\right)$ be a finite number of points on $M$. Let $k=\left(k_{1}, \ldots, k_{n}, \varepsilon\right)$ denote a vector where $k_{i}$ is a positive integer, $\sum k_{i}=4 g-4$, and $\varepsilon= \pm 1$. Let $Q_{k}$ denote the space of holomorphic quadratic differentials $q$ on $M$ with zeros at $x_{1}, \ldots, x_{n}$ with orders $k_{1}, \ldots, k_{n}$; given a + sign if $q$ is the square of an abelian differential, a - sign otherwise. 
Note $\int|q|$ defines an area element and $\left|q^{1 / 2} d z\right|$ a line element. Let $Q_{k}^{0}$ be the corresponding subspace of quadratic differentials with unit area. Following Veech [V2], we call $Q_{k}^{0}$ a stratum inside the space of all quadratic differentials. Let Map be the mapping class group of $(M, \Sigma)$. It acts properly discontinuously on $Q_{k}^{0}$.

Now given $q \in Q_{k}^{0}$ and $t \in \mathbf{R}$ consider the Teichmüller map with dilatation

$$
\frac{e^{t}-1}{e^{t}+1} \bar{q} /|q| \text {. }
$$

The terminal quadratic differential is also in $Q_{k}^{0}$, that is, Teichmüller maps preserve orders of zeros and orientability. Thus the Teichmüller geodesic flow preserves $Q_{k}^{0}$ and is preserved by the action of Map. Thus we have a flow on each $Q_{k}^{0} /$ Map which we denote by $g_{t}$. We will also simply write $q_{t}$ for $g_{t}(q)$. The topology on $Q_{k}^{0} /$ Map is such that a geodesic diverges if and only if it eventually leaves every compact set.

Theorem 1. There is a $\delta_{0}>0$ such that the set of $q \in Q_{k}^{0}$ such that $g_{t}(q)$ is a divergent geodesic has Hausdorff dimension $\operatorname{dim} Q_{k}^{0}-1+\delta_{0}$.

Remarks. First it was shown [M1] that the space of all quadratic differentials over Teichmüller space has a finite measure invariant under the flow and the flow is ergodic. This was extended by Veech [V2] to a measure $\lambda$ and ergodicity on each $Q_{k}^{0} /$ Map . Ergodicity implies the set of quadratic differentials defined in Theorem 1 has measure zero.

For $k \neq(4 g-4, \pm)$, zeros of $q \in Q_{k}^{0}$ may coalesce at "infinity" to higher order zeros. On the other hand, for $k=(4 g-4, \pm)$ the underlying Riemann surfaces on a divergent geodesic will leave every compact set of the underlying moduli space of Riemann surfaces.

We actually prove a stronger theorem that implies Theorem 1 . We start with some notation.

A $\theta$ trajectory of $q$ is an arc along which $\arg q(z) d z^{2}=\theta$. Horizontal trajectories correspond to $\theta=0$ and vertical trajectories to $\theta=\pi$. A saddle connection $\beta$ is a trajectory joining two zeros of $q$. By $\theta_{\beta}$, the vertical angle of $\beta$, we mean the angle $\theta$ for which $\beta$ is a vertical trajectory of $e^{i \theta} q$. We say two saddle connections $\beta$ and $\gamma$ are disjoint if they meet at most at common zeros. Now orient $\beta$ and $\gamma$ and choose a branch of $q^{1 / 2} d z$ along each. Define

$$
|\beta \times \gamma|=\left|\int_{\beta} \operatorname{Re} q^{1 / 2} d z \int_{\gamma} \operatorname{Im} q^{1 / 2} d z-\int_{\gamma} \operatorname{Re} q^{1 / 2} d z \int_{\beta} \operatorname{Im} q^{1 / 2} d z\right| .
$$

It is not difficult to see the definition does not depend on the orientation or the choice of branch. It is also clear that $|\beta \times \gamma|$ is invariant under $q \rightarrow e^{i \theta} q$ and $q \rightarrow q_{t}$.

Now for a given $q \in Q_{k}^{0}$ consider the family of geodesics $g_{t}\left(e^{i \theta} q\right)$ where $0 \leq \theta \leq 2 \pi$. This is the so-called Teichmüller disc. It is not difficult to see that there are only countably many values of $\theta$ for which $e^{i \theta} q$ has a vertical 
saddle connection $\beta$. These are precisely the values of $\theta$ for which $g_{t}\left(e^{i \theta} q\right)$ is a pinching geodesic as the length of $\beta$ goes to zero since Teichmüller maps contract in the vertical direction.

In addition it was shown in [KMS] that the set of $\theta$ for which $g_{t}\left(e^{i \theta} q\right)$ is a divergent geodesic has Lebesgue measure zero. This was used to show that for a.e. $\theta$ the vertical foliation of $e^{i \theta} q$ is uniquely ergodic. Katok asked whether the Hausdorff dimension of the divergent geodesics is zero. We show that for generic $q$ it is positive and formulate such a generic condition which is sufficiently weak so that it holds for a.e. $q$ in $Q_{k}^{0}$. Then Theorem 1 will follow from Theorem 2.

Theorem 2. There exists $\delta_{0}>0$ so that for $\lambda$ a.e. $q \in Q_{k}^{0},\left\{\theta: g_{t}\left(e^{i \theta} q\right)\right.$ is a divergent geodesic $\}$ has Hausdorff dimension $\delta_{0}$.

Remark. Veech [V3] has found a dense set of $q$ so that $g_{t}\left(e^{i \theta} q\right)$ does not spiral for any $\theta$.

We will find a set of $q$ of full measure for which one can make the following construction. For any $q$ in this set and saddle connection $\beta$ of $q$ and for any positive integer $n$ we will find an interval $I(\beta, n)$ centered at the vertical angle $\theta_{\beta}$. For each $\theta \in I(\beta, n)$ there will be an interval $J(\beta, n, \theta)$ of times $t$ such that the $g_{t}\left(e^{i \theta} q\right)$ length of $\beta$ is less than $1 / n$ for $t \in J(\beta, n, \theta)$.

We will find a set $\gamma_{1}, \ldots, \gamma_{r}$ of saddle connections with certain lengths with the properties that

(i) $I\left(\gamma_{i}, n+1\right) \subset I(\beta, n)$,

(ii) $I\left(\gamma_{i}, n+1\right) \cap I\left(\gamma_{j}, n+1\right)=\varnothing, i \neq j$, and

(iii) $J\left(\gamma_{i}, n+1, \theta\right) \cap J(\beta, n, \theta) \neq \varnothing$.

Condition (i) means the interval of angles for which $\gamma_{i}$ is short for some interval of time $J$ is contained in those for which $\beta$ is short for some time. Condition (iii) means there is a common period of time when both $\beta$ and $\gamma_{i}$ are short. Thus for a connected interval of times, the union of the two intervals $J$, either one or both are short. For this set of full measure we will then iterate this procedure to find further intervals inside each $I\left(\gamma_{i}, n+1\right)$ with further overlapping intervals of times $J$. For any point in the set we will have a sequence of saddle connections such that for any sufficiently large time there is at least one which is arbitrarily short.

This will construct the Cantor set as an infinite intersection of intervals. The Hausdorff dimension will be estimated by controlling the size of the interval $I(\beta, n)$, their spacing, and the number $r(\beta)$ of intervals inside each. In fact we will have

$$
|I(\beta, n)|=\sigma^{p^{n}} \quad \text { for constants } \sigma<1, p>1
$$

so the size of the intervals will decrease super exponentially. On the other hand we will find $r(\beta)=\sigma^{-s p^{n}}$ for $s>0$ so the number of intervals inside $I(\beta, n)$ will grow super exponentially as well. This will show the Hausdorff dimension is positive almost everywhere. Ergodicity of the flow will imply it is constant. 
This paper is organized as follows. In $\S 1$ we will consider the Hausdorff dimension of certain Cantor sets. In $\S 2$ we will state the main Proposition 2.3 on the existence of saddle connections $\gamma_{1}, \ldots, \gamma_{r}$ disjoint from a given $\beta$ and use it to define the Cantor set as a decreasing sequence of closed sets. In $\S 3$ we will prove this main proposition. In $\S 4$ we will show the construction is possible for a.e. $q$.

We assume the reader is familiar with the basic notion of a quadratic differential and Teichmüller map. Basic references are [Ber, G, S].

We would like to express gratitude to A. Katok for valuable conversations.

\section{HAUSdORFF Dimension OF CERTAIN CANTOR SETS ON $[0,1]$}

Recall first the definition of $\delta$ Hausdorff outer measure. Given $E \subset \mathbf{R}^{n}$ and $\varepsilon>0$ let $H_{\delta, \varepsilon}(E)=\inf _{\mathscr{B}} \sum_{B \in \mathscr{B}}\left(r_{B}\right)^{\delta}$, where $\mathscr{B}$ is a cover of $E$ by balls $B$ of radius $r_{B}<\varepsilon$. The $\delta$ outer measure of $E$ is defined as $H_{\delta}(E)=$ $\lim _{\varepsilon \rightarrow 0} H_{\delta, \varepsilon}(E)$. The Hausdorff dimension of $E$ is the sup of those $\delta$ for which $H_{\delta}(E)=\infty$.

Suppose $C \subset[0,1]$ is a Cantor set constructed as a decreasing intersection of closed sets $C_{n}$ each of which is a disjoint union of closed intervals $\Delta_{n}$ called the level $n$ sets. We suppose there are constants $k_{2}>k_{1}>0$ such that

(1) Each level $n$ set $\Delta_{n}$ contains at least $\left|\Delta_{n}\right|^{-k_{1}}$ level $n+1$ subsets.

(2) The sizes of the level $n+1$ subsets are at least $\left|\Delta_{n}\right|^{k_{2}}$.

(3) The gap between any level $n+1$ subsets is at least $\left|\Delta_{n}\right|^{k_{2}}$.

Proposition 1.1. Under the above hypotheses, let $\rho=k_{1} / 2\left(k_{2}-k_{1}\right) k_{2}$. Then $H^{\rho}(C) \neq 0$ so $\operatorname{dim} C \geq \rho$.

We first prove

Lemma 1.2. Suppose $U_{1}, \ldots, U_{p}$ is a cover of $\Delta_{n} \cap C$ for some level $n$ set $\Delta_{n}$ such that at least half of the level $n+1$ subsets of $\Delta_{n}$ have the property that both it and a neighbor are intersected by the same $U_{j}$. Then for any $0<\sigma<1$

$$
\sum_{i=1}^{p}\left|U_{j}\right|^{\sigma} \geq \frac{1}{4} \sigma\left|\Delta_{n}\right|^{\sigma\left(k_{2}-k_{1}\right)} \text {. }
$$

Proof. If a $U_{j}$ intersects two or more level $n+1$ subsets, its linear measure is at least as big as the sum of the gaps between them which by (3) is at least $\left|\Delta_{n}\right|^{k_{2}}$ multiplied by the number of gaps covered. The number of gaps covered is at least $\frac{1}{2}$ the number of sets with the property that both it and a neighbor are intersected by the same $U_{j}$. This in turn is at least $\frac{1}{2}$ the total number of sets by hypothesis. This gives $\sum_{i=1}^{p}\left|U_{i}\right| \geq \frac{1}{4}\left|\Delta_{n}\right|^{k_{2}-k_{1}}$.

The lemma follows since the function $f(x)=x^{\sigma}$ is concave for $\sigma<1$.

Proof of Proposition 1.1. Suppose $H^{\rho}(C)=0$. We will arrive at a contradiction. Let $\delta_{m}$ be the maximum linear size of any level $m$ set. By (2) $\lim _{m \rightarrow \infty} \delta_{m}=0$. 
Choose $K$ large enough so that for $m \geq K-1$

$$
\frac{1}{2}\left(\frac{1}{4}\right)^{\rho} \delta_{m}^{\rho\left(k_{2}-k_{1}\right) k_{2}-k_{1}} \geq 1 .
$$

This is possible since $\rho\left(k_{2}-k_{1}\right) k_{2}-k_{1}=-k_{1} / 2<0$. Now if $H^{\rho}(C)=0$, for any $0<\varepsilon<1$ there is a finite cover $U_{1}, \ldots, U_{p}$ of $C$ with

(a) $\left|U_{i}\right|<\min _{\Delta_{K-1}}\left|\Delta_{K-1}\right|^{k_{2}}$.

(b) $\sum\left|U_{i}\right|^{\rho}<\varepsilon$.

Now (3) and (a) imply each $U_{i}$ intersects at most one level $K$ set. Let $n_{i} \geq K$ be the largest integer such that $U_{i}$ intersects only one level $n_{i}$ set. Since $\lim _{m \rightarrow \infty} \delta_{m}=0$, this number is well defined. Let $N=\max n_{i}$ and $F_{n}=\left\{\Delta_{n}\right.$ : for any $U_{i}$ such that $U_{i} \cap \Delta_{n} \neq \varnothing, U_{i} \cap \Delta_{n}^{\prime}=\varnothing$ for all $\left.\Delta_{n}^{\prime} \neq \Delta_{n}\right\}$. Then $F_{N+1}=\varnothing$ and all level $K$ sets are in $F_{K}$. Let $J, K \leq J \leq N$, be the number such that for some $\Delta_{J-1}$, at least half of its level $J$ subsets are in $F_{J}$ and there is no $j>J$ with the same property. Let $\Delta_{J-1}$ be a level $J-1$ set with the above property. Let $\delta=\left|\Delta_{J-1}\right|$. Then by (1) and the definition, $\Delta_{J-1}$ contains at least $\frac{1}{2} \delta^{-k_{1}}$ level $J$ subsets $\Delta_{J}$ which are in $F_{J}$. For each $\Delta_{J} \in F_{J}$ at least half of its level $J+1$ subsets are not in $F_{J+1}$ since $J$ is maximal. Then for each such $\Delta_{J} \in F_{J}$, associate to it the set of $U_{i}$ which intersect it. By the definition of $F_{J}$ the $U_{i}$ associated to different $\Delta_{j} \in F_{J}$ are distinct. For any such $\Delta_{J} \in F_{J}$, by the lemma $\sum\left|U_{i}\right|^{\rho} \geq \frac{1}{4}\left|\Delta_{J}\right|^{\rho\left(k_{2}-k_{1}\right)}$, the sum over the $U_{i}$ associated to $\Delta_{J}$. Since the $U_{i}$ associated to different $\Delta_{J}$ are distinct,

$$
\sum_{i=1}^{p}\left|U_{i}\right|^{\rho} \geq \sum_{\Delta_{j} \in F_{J}}\left(\frac{1}{4}\right)^{\rho}\left|\Delta_{J}\right|^{\rho\left(k_{2}-k_{1}\right)} .
$$

By (2), $\left|\Delta_{J}\right| \geq \delta^{k_{2}}$. Since there are at least $\frac{1}{2} \delta^{-k_{1}}$ such $\Delta_{J}$,

$$
\sum_{i=1}^{p}\left|U_{i}\right|^{\rho} \geq \frac{1}{2}\left(\frac{1}{4}\right)^{\rho} \delta^{\rho k_{2}\left(k_{2}-k_{1}\right)} \geq \frac{1}{2}\left(\frac{1}{4}\right)^{\rho} \delta_{J-1}^{\rho k_{2}\left(k_{2}-k_{1}\right)},
$$

the last inequality since $\delta_{J-1} \geq \delta$ and the exponent is negative. But the last quantity $\geq 1$ and so $\sum_{i=1}^{p}\left|U_{i}\right|^{\rho} \geq 1$, a contradiction.

\section{Construction of the CANTOR SET}

We begin with some notation. For any $q$, saddle connection $\beta$, and $\theta$ let

$$
h_{\theta}(\beta)=\int_{\beta}\left|\operatorname{Re}\left(e^{i \theta} q\right)^{1 / 2} d z\right| \text { and } v_{\theta}(\beta)=\int_{\beta}\left|\operatorname{Im}\left(e^{i \theta} q\right)^{1 / 2} d z\right|
$$

be the horizontal and vertical lengths of $\beta$, respectively. If $\theta=0$ we simply write $h(\beta)$ and $v(\beta)$. Let $|\beta|=\left(h_{\theta}(\beta)^{2}+v_{\theta}(\beta)^{2}\right)^{1 / 2}$ be the $e^{i \theta} q$-length of $\beta$. It is clearly independent of $\theta$. We will also denote by $v_{\theta, t}(\beta), h_{\theta, t}(\beta)$, and $|\beta|_{\theta, t}$ the corresponding lengths with respect to $\left(e^{i \theta} q\right)_{t}$. Again when $\theta=0$ 
we delete it. We have the following formulas which are a consequence of the definition of the Teichmüller map:

$$
v_{\theta, t}(\beta)=e^{-t / 2} v_{\theta}(\beta), \quad h_{\theta, t}(\beta)=e^{t / 2} h_{\theta}(\beta) .
$$

We will define an interval of angles $I$ and an interval of times $J$. Suppose $\beta$ is a saddle connection and $n$ is an integer. For any $t_{0}$ such that

$$
e^{t_{0} / 2} \geq 2 n|\beta|
$$

let

$$
I=I\left(\beta, n, t_{0}\right)=\left\{\theta:\left|\frac{\sin \left(\theta-\theta_{\beta}\right)}{2}\right| \leq \frac{e^{-t_{0} / 2}}{n|\beta|}\right\} .
$$

For $\theta \in I$, let

$$
J=J(\beta, n, \theta)=\left\{t: \sqrt{2} n|\beta| \leq e^{t / 2} \leq \frac{1}{\sqrt{2} n|\beta|\left|\sin \left(\theta-\theta_{\beta}\right) / 2\right|}\right\} .
$$

Lemma 2.1. $|\beta|_{\theta, t} \leq 1 / n$ for any $\theta \in I$ and $t \in J$.

Proof. $\left|\sin \left(\left(\theta-\theta_{\beta}\right) / 2\right)\right|=h_{\theta}(\beta) /|\beta|$ so $h_{\theta}(\beta)=|\beta|\left|\sin \left(\left(\theta-\theta_{\beta}\right) / 2\right)\right|$. Then $e^{t / 2} h_{\theta}(\beta) \leq 1 / \sqrt{2} n$. Further $v_{\theta}(\beta) \leq|\beta|$ so $e^{-t / 2} v_{\theta}(\beta) \leq 1 / \sqrt{2} n$ for $e^{t / 2} \geq$ $\sqrt{2} n|\beta|$.

Lemma 2.2. With the same notation as in Lemma 2.1 let $\gamma$ be a saddle connection and let $t_{1}$ exist such that

(a) $|\gamma|\left|\sin \left(\left(\theta_{\gamma}-\theta_{\beta}\right) / 2\right)\right| \leq 1 / 2|\beta|(n+1)^{2}$.

(b) $e^{t_{1} / 2} \geq \max (2(n+1)|\gamma|, 2(n+1) n|\beta|)$.

(c) $I\left(\gamma, n+1, t_{1}\right) \subset I\left(\beta, n, t_{0}\right)$.

Then for $\theta \in I\left(\gamma, n+1, t_{1}\right)$ we have $J(\beta, n, \theta) \cap J(\gamma, n+1, \theta) \neq \varnothing$ and for $t \in J(\beta, n, \theta) \cup J(\gamma, n+1, \theta)$ either $|\beta|_{\theta, t} \leq 1 / n$ or $|\gamma|_{\theta, t} \leq 1 /(n+1)$.

Proof. For $\theta \in I\left(\gamma, n+1, t_{1}\right)$

$$
\begin{aligned}
\left|\sin \frac{\left(\theta-\theta_{\beta}\right)}{2}\right| & \leq\left|\sin \frac{\left(\theta-\theta_{\gamma}\right)}{2}\right|+\left|\sin \frac{\left(\theta_{\gamma}-\theta_{\beta}\right)}{2}\right| \\
& \leq \frac{e^{-t_{1} / 2}}{|\gamma|(n+1)}+\frac{1}{2|\gamma||\beta|(n+1)^{2}} \leq \frac{1}{2(n)(n+1)|\beta||\gamma|}
\end{aligned}
$$

by (a) and (b). Thus

$$
\sqrt{2}(n+1)|\gamma| \leq \frac{1}{\sqrt{2} n|\beta|\left|\sin \left(\left(\theta-\theta_{\beta}\right) / 2\right)\right|} .
$$

This means $J(\gamma, n+1, \theta) \cap J(\beta, n) \neq \varnothing$. The second statement in the lemma is merely a restatement of Lemma 2.1 .

Now the construction of the Cantor set is based on the following proposition whose proof will occupy $\S 3$. 
Fix $j>0$. For $\beta$ a saddle connection of $q$, we say $q \in F_{j}(\beta)$ if either

(i) $\beta$ is part of or equal to one boundary component of an annulus of closed $\theta$ trajectories such that the complement of the annulus has area $\leq 1 /|\beta|^{j}$ or,

(ii) there is a subsurface with boundary containing $\beta$ in its interior with area $\leq 1 /|\beta|^{j}$ and such that the boundary consists of saddle connections $\gamma$ with $|\gamma| \leq|\beta|$.

Remark. The definition means there is a $\gamma$ of smaller length and almost in the same direction as $\beta$ or there is an annulus whose complement has small area.

Let $F_{j}=\lim _{\beta} \sup F_{j}(\beta)=\left\{q: q\right.$ belongs to $F_{j}(\beta)$ for infinitely many $\left.\beta\right\}$.

We will show in Proposition 4.2 that $F_{j}$ has zero measure for $j$ sufficiently large.

Now suppose $q \notin F_{j}$. Since there are only finitely many $\beta$ such that $q \in F_{j}(\beta)$ choose an interval $I$ so $q \notin F_{j}(\beta)$ for any $\beta$ such that $\theta_{\beta} \in I$. Choose $I^{\prime} \subset I$.

Proposition 2.3. There exist constants $K^{\prime}>1$ and $0<r_{1}, r_{2}<1$ with the following property.

Suppose $\beta$ is a saddle connection and $k, n$ are such that $|\beta| \geq n^{2} \geq 4$, $k>K^{\prime}$, and $I(\beta, n, 2 k \log |\beta|) \subset I^{\prime}$. Then for all $\theta \in I(\beta, n, 2 k \log |\beta|)$ there is a saddle connection $\gamma$ disjoint from $\beta$ such that

(i) $\theta_{\gamma} \in I(\beta, n, 2 k \log |\beta|)$,

(ii) $|\beta|^{2} \leq\left(|\beta|^{k}\right)^{r_{1}} \leq|\gamma| \leq|\beta|^{k} / 2(n+1)^{2}$,

(iii) $|\gamma|\left|\sin \left(\left(\theta_{\gamma}-\theta_{\beta}\right) / 2\right)\right| \leq 1 / 2|\beta|(n+1)^{2}$, and

(iv) $\left|\left(\theta-\theta_{\gamma}\right) / 2\right| \leq 2\left|\sin \left(\left(\theta-\theta_{\gamma}\right) / 2\right)\right| \leq|I(\beta, n, 2 k \log |\beta|)|^{1+r_{2}}$.

Remark. (iv) says for any $\theta$ in the interval $I$ there is a $\theta_{\gamma}$ quite near to it, so there will be many such $\theta_{\gamma}$. (ii) controls the length of $\gamma$ which inductively will control the sizes of the next set of intervals. (iii) is the condition needed in Lemma 2.1 to allow $\beta$ and $\gamma$ to be made simultaneously short. This will altogether allow the interval $I$ defined by $\beta$ to give rise to subintervals defined by $\gamma$.

In the remainder of this section we show how this proposition allows us to build a Cantor set of divergent geodesics of positive Hausdorff dimension.

First choose $s>0$ so $x^{s} \geq 3 x^{r_{2}}$ for $x \geq 2$, where $r_{2}$ was given by Proposition 2.3. Suppose now $q \in E=Q_{k}^{0}-F_{j}$. There is a finite set $B$ of $\gamma$ such that $q \in F_{j}(\gamma)$. Choose $\beta_{0}$ not in this set with large enough length $T_{0}$ such that $I_{1}=I\left(\beta_{0}, 1,2 K^{\prime} \log T_{0}\right)$ does not contain any $\theta_{\gamma}$ for $\gamma \in B$.

$I_{1}$ is our starting level 1 set $\Delta_{1}$. We wish inductively to construct sets $\Delta_{n}$, $n \geq 1$, satisfying the hypotheses of Lemma 1.2. Let $p=K^{\prime}+2$. Each $\Delta_{n}$ will have size $\left(1 / T_{0}\right)^{p^{n}}$. We further require the center of $\Delta_{n}$ to be $\theta_{\beta}$ for a saddle 
connection $\beta$ such that

$$
n^{2} \leq|\beta| \leq T_{0}^{p^{n-1}}
$$

We will also associate a time $t$ satisfying (2.2). Of course $\Delta_{1}$ satisfies all hypotheses. Now suppose inductively we have found $\Delta_{n}$ centered at $\theta_{\beta}$, which by rotating we can assume to be 0 . Now $q \notin F_{j}(\beta)$. Choose $t=t(\beta, n)$ so

$$
\left|\Delta_{n}\right|=|I(\beta, n, t)|=1 / T_{0}^{p^{n}} \text {. }
$$

Now $I(\beta, n, t(\beta, n))=\left\{\theta:|\sin (\theta / 2)| \leq e^{-t / 2} /|\beta| n\right\}$ so (2.3) and (2.4) imply

$$
e^{t / 2} \geq \frac{1}{n} T_{0}^{p^{n}-p^{n-1}} \geq\left(T_{0}^{p^{n-1}}\right)^{K^{\prime}} \geq \max \left(|\beta|^{K^{\prime}}, 2 n|\beta|\right) .
$$

Now define $k$ by $|\beta|^{k}=e^{t / 2}$ so $(2.5)$ implies $k \geq K^{\prime}$. Decompose $\Delta_{n}=$ $I(\beta, n, t)$ into $\left|\Delta_{n}\right|^{-r_{2}}$ disjoint intervals of width $\left|\Delta_{n}\right|^{1+r_{2}}$.

By Proposition 2.3, ignoring the end intervals, each such interval contains $\theta_{\gamma}$ for some saddle connection $\gamma$ with $|\gamma|$ satisfying

$$
(n+1)^{2} \leq|\beta|^{k r_{1}} \leq|\gamma| \leq|\beta|^{k r_{1}} / 2(n+1)^{2} \leq T_{0}^{p^{n}} / 2(n+1)^{2}
$$

and

$$
|\gamma|\left|\sin \frac{\theta_{\gamma}}{2}\right| \leq \frac{1}{2|\beta|(n+1)^{2}} .
$$

There are at least $1 /\left|\Delta_{n}\right|^{r_{2}}$ such $\gamma$. For each $\gamma$ let $t_{1}=t_{1}(\gamma)$ satisfy

$$
\left|\left\{\theta:\left|\sin \frac{\left(\theta-\theta_{\gamma}\right)}{2}\right| \leq \frac{e^{-t_{1} / 2}}{|\gamma|(n+1)}\right\}\right|=\frac{1}{T_{0}^{p^{n+1}}} .
$$

Then (2.6) and (2.8) give

$$
\begin{aligned}
e^{t_{1} / 2} & \geq \frac{T_{0}^{p^{n}+1} 2(n+1)^{2}}{(n+1) T_{0}^{p^{n}}}=2(n+1) T_{0}^{p^{n+1}-p^{n}} \\
& \geq \max (2(n+1)|\gamma|, 2(n+1) n|\beta|),
\end{aligned}
$$

so (2.2) is satisfied. Next by eliminating at most half of the $\gamma$ we can guarantee any two $\theta_{\gamma}$ are at least $\frac{1}{2}|I|^{1+r_{2}}$ apart. About each remaining $\gamma$ we take an interval $\Delta_{n+1}=I\left(\gamma, n+1, t_{1}\right)$ which has length $\left|\Delta_{n+1}\right|=1 / T_{0}^{p^{n+1}}=\left|\Delta_{n}\right|^{p}$. Since $p>1+r_{2}$, their distance apart is larger than their size. By removing possibly the $\theta_{\gamma}$ closest to the endpoints, each $\Delta_{n+1} \subset \Delta_{n}$. This completes the induction step.

Now for each $\theta \in \Delta_{n+1}=I\left(\gamma, n+1, t_{1}(\gamma)\right)$ by Lemma 2.2, (2.5), and (2.7) there is a connected interval of times $J(\beta, n, \theta) \cup J(\gamma, n+1, \theta)$ for which either $\beta$ or $\gamma$ has length $\leq \frac{1}{n}$. The Cantor set $C$ consists of all $\theta$ which are in infinitely many $\Delta_{n}$. For $\theta \in C$ there is a sequence of $\beta_{0}, \ldots, \gamma_{n}, \gamma_{n+1}, \ldots$ of saddle connections and a connected union of intervals

$$
J\left(\gamma_{n}, n, \theta\right) \cup J\left(\gamma_{n+1}, n+1, \theta\right) \cup \cdots
$$


whose union is $\left\{t: t_{0} \leq t<\infty\right\}$ such that for any $N$ there is a $t_{N}$ so that for all $t \geq t_{N}$ there is a $\gamma_{j}$ with $\left|\gamma_{i}\right|_{\theta, t} \leq \frac{1}{N}$. This shows each $\theta \in C$ defines a divergent geodesic. By Theorem $1.1 C$ has a Hausdorff $\operatorname{dim} \geq s / 2 p^{2}>0$. Thus we have proved (assuming Proposition 2.3)

Proposition 2.4. There is a $\delta=\delta(j)>0$ such that for each $q \notin F_{j}$ there is a Cantor set $C$ in $[0,2 \pi]$ of Hausdorff $\operatorname{dim}>\delta$ such that for each $\theta \in C$, $\left(e^{i \theta} q\right)_{t}$ is a divergent geodesic.

\section{FINDING SADDLE CONNECTIONS}

In this section we will prove Proposition 2.3. The idea is to find a horizontal trajectory for $e^{i \theta} q$, use it to decompose the surface into rectangles (cf. [S]) with horizontal sides on the trajectory, and find saddle connections by joining zeros on opposite sides of the rectangle.

We first need some additional notation and a result from [M3]. A subcomplex $Y$ of a surface is a triangulation of part of the surface such that the vertices are zeros of $q$, edges are saddle connections, and faces are triangles with no zeros in their interior. A system $\Gamma=\left(\gamma_{1}, \ldots, \gamma_{p}\right)$ of mutually disjoint saddle connections is on the boundary of $Y$ if each $\gamma_{i}$ bounds fewer than two triangles in $Y$.

Proposition 3.1 [M3, Proposition 1.7]. Suppose $Y$ is a complex with boundary $\Gamma$, and $q$ is a quadratic differential of unit area on $Y$. Suppose $\beta$ is a saddle connection contained in $Y$, not necessarily an edge, and $|\beta| \geq 2|\gamma|$ for all $\gamma \in \Gamma$. Then there is a number $D>1$ such that either $|\beta \times \gamma| \leq D$ for each $\gamma \in \Gamma$ or there is a subcomplex $Z \subset Y$ with boundary $\Omega$ such that $|\beta| \geq 4|\omega|$ and $|\beta \times \omega| \leq D$ for all $\omega \in \Omega$.

The number $D$ will be referred to in the rest of this section. Next we investigate conditions under which we can find horizontal trajectories of a given size disjoint from $\beta$.

Proposition 3.2. Fix $j$. There exist $K=K(j)$ and $\alpha=\alpha(D, g)>0$ with the following property. Suppose $\beta$ is a saddle connection of $q$. Assume

(i) If $\beta$ is part of one boundary component of an annulus of closed trajectories, the complement of the annulus has area at least $1 /|\beta|^{j}$.

(ii) $\beta$ is not contained in a complex $Y \subset X$ with boundary $\Gamma$ such that $|\gamma| \leq|\beta| / 2$ for all $\gamma \in \Gamma$ and area $Y \leq 1 / D|\beta|^{j}$.

Then for any $L \geq|\beta|^{K}$ and $\theta$ such that for $\left|\sin \left(\left(\theta-\theta_{\beta}\right) / 2\right)\right| \leq 1 / L|\beta|$ there is a horizontal trajectory $h$ of $e^{i \theta} q$ of length $\alpha / D L$ with one endpoint a zero of $q$ such that $h$ is disjoint from $\beta$ except possibly for a common zero and if $\beta$ is part of one boundary component of an annulus, then $h$ is disjoint from the annulus. 
Proof. Suppose the proposition is false. Then there exists a sequence $\beta_{n}$ of saddle connections (of a variable $q, X$ ) and sequences $\alpha_{n}, \theta_{n}, K_{n}, L_{n}$ such that

(a) $\alpha_{n} \rightarrow 0$,

(b) $K_{n} \rightarrow \infty$,

(c) $L_{n} \geq\left|\beta_{n}\right|^{K_{n}}$,

(d) $\left|\sin \left(\left(\theta_{n}-\theta_{\beta_{n}}\right) / 2\right)\right| \leq 1 / L_{n}\left|\beta_{n}\right|$, and

(e) there is no horizontal segment of $e^{i \theta_{n}} q$ of length $\alpha_{n} / D L_{n}$ disjoint from $\beta_{n}$.

By (d) for some $e^{t_{n} / 2} \leq\left|\beta_{n}\right|$ we have

$$
e^{-t_{n} / 2} v_{\theta_{n}}\left(\beta_{n}\right)=1 ; \quad e^{t_{n} / 2} h_{\theta_{n}}\left(\beta_{n}\right) \leq\left|\beta_{n}\right| / L_{n} \leq 1 .
$$

Consider the Teichmüller map with dilatation

$$
\frac{e^{t_{n}}-1}{e^{t_{n}}+1} e^{i \theta_{n}} q
$$

let $X_{n}$ be the image surface, and let $q_{n}=g_{t_{n}}\left(e^{i \theta_{n}} q\right)$ be the terminal quadratic differential. By passing to subsequences we can assume $X_{n} \rightarrow X_{\infty}$ and $q_{n} \rightarrow$ $q_{\infty}$, where $X_{\infty}$ is compact except possibly for punctures.

Case I. $X_{\infty}$ is compact. Now $\left\|q_{\infty}\right\|=\left\|q_{n}\right\|=1$. Since $\beta_{n}$ has length $\leq 2$ on $X_{n}$ we may assume $\beta_{n} \rightarrow \beta_{\infty}$ where $\left|\beta_{\infty}\right| \leq 2$. Choose a horizontal trajectory of some positive length $2 \alpha / D$ on $X_{\infty}$ disjoint from $\beta_{\infty}$ and with one endpoint a zero of $q_{\infty}$. Then for $X_{n}$ near $X_{\infty}$ there is a corresponding horizontal trajectory of length at least $\alpha / D$ disjoint from $\beta_{n}$. Mapping back to $X$ by the inverse Teichmüller map gives a horizontal trajectory of length at least

for $n$ big enough, a contradiction.

$$
\frac{e^{-t_{n} / 2} \alpha}{D} \geq \frac{\alpha_{n}}{D\left|\beta_{n}\right|} \geq \frac{\alpha_{n}}{D L_{n}}
$$

Case II. $X_{\infty}$ has punctures so one or more homotopy classes of simple closed curves have been pinched. If $q_{\infty} \neq 0$ on some component of $X_{\infty}$, the proof proceeds much as in the first case. If $\beta_{n}$ does not intersect that component, we can find a horizontal trajectory of length $2 \alpha / D$ on $X_{\infty}$ in that component. For large $n$ we can find a horizontal trajectory of length $\alpha / D$ on $X_{n}$ disjoint from $\beta_{n}$ and again we get a contradiction. If $\beta_{n}$ intersects the component on which $q_{\infty} \neq 0$, we may assume again $\beta_{n}$, intersected with that component, converges to a saddle connection $\beta_{\infty}$ of bounded length. Again there is a horizontal trajectory of length $2 \alpha / D$ disjoint from $\beta_{\infty}$ and horizontal trajectory of length $\alpha / D$ disjoint from $\beta_{n}$ and we proceed as above.

Thus we may assume $q_{\infty} \equiv 0$. This implies [M1] that $q_{n}$ has closed trajectories in some homotopy class being pinched and any curve crossing the annulus has length $\rightarrow \infty$. Since the length of $\beta_{n}$ is bounded, $\beta_{n}$ does not 
cross the annulus. Thus $\beta_{n}$ is contained in a complex with boundary whose length $\rightarrow 0$. By taking smaller surfaces with boundary if necessary, we may assume there is a smallest complex $Z_{n}$ containing $\beta_{n}$ with boundary $\Omega_{n}$ such that $\left|\Omega_{n}\right|_{\theta_{n}, t_{n}} \leq|\beta|_{\theta_{n}, t_{n}} / 4 . Z_{n}$ is smallest in the sense there is no subcomplex of $Z_{n}$ with the same properties.

Since $h_{\theta_{n}, t_{n}}\left(\beta_{n}\right) \leq v_{\theta_{n}, t_{n}}\left(\beta_{n}\right)$ and $\left|\Omega_{n}\right|_{\theta_{n}, t_{n}} \leq\left|\beta_{n}\right|_{\theta_{n}, t_{n}} / 4$,

$$
\max \left(v_{\theta_{n}, t_{n}}\left(\Omega_{n}\right), h_{\theta_{n}, t_{n}}\left(\Omega_{n}\right)\right) \leq v_{\theta_{n}, t_{n}}\left(\beta_{n}\right) / 2 \sqrt{2}
$$

which implies

$$
\left|\Omega_{n}\right| \leq v\left(\beta_{n}\right) / 2 \leq\left|\beta_{n}\right| / 2,
$$

with the lengths measured on $X$.

Now let $\delta_{n}=$ area $Z_{n}$. By assumption (ii)

$$
\delta_{n} \geq 1 / D\left|\beta_{n}\right|^{j} .
$$

Let $\tilde{q}_{n}=q_{n} / \delta_{n}$. Then $Z_{n}$ has $\tilde{q}_{n}$-area 1 . Then the $\tilde{q}_{n}$ vertical and horizontal lengths of $\beta_{n}$ are

$$
1 / \delta_{n}^{1 / 2} \text { and } \leq\left|\beta_{n}\right| / L_{n} \delta_{n}^{1 / 2},
$$

respectively. Now by Proposition 3.1 we can assume $\left|\beta_{n} \times \omega_{n}\right| \leq D$ for each $\omega_{n} \in \Omega_{n}$, with the cross product measured with respect to $\tilde{q}_{n}$. For if not, by Proposition 3.1 we could find a smaller complex $Z_{n}^{\prime}$ containing $\beta_{n}$ with boundary $\Omega_{n}^{\prime}$ such that $\left|\omega_{n}^{\prime}\right| \leq\left|\beta_{n}\right| / 4$ for each $\omega_{n}^{\prime} \in \Omega_{n}^{\prime}$, a contradiction to $Z_{n}$ being the smallest such complex containing $\beta_{n}$.

Once more we contract the vertical length of $\beta_{n}$ with a Teichmüller map with dilatation

$$
\frac{e^{t_{n}^{\prime}}-1}{e^{t^{\prime} n}+1} \tilde{q}_{n} /\left|\tilde{q}_{n}\right|, \quad e^{t_{n}^{\prime} / 2}=\frac{1}{\delta_{n}^{1 / 2}} .
$$

Call $Z_{n}^{\prime}$ the image of $Z_{n}$ and $\tilde{q}_{n}^{\prime}$ the terminal quadratic differential under this Teichmüller map. By (3.3) and (3.4) the new horizontal length of $\beta_{n}$ is

$$
\leq \frac{\left|\beta_{n}\right|}{L_{n} \delta_{n}^{1 / 2} \delta_{n}^{1 / 2}} \leq \frac{\left|\beta_{n}\right|^{j+1}}{L_{n}} .
$$

Since $L_{n} \geq\left|\beta_{n}\right|^{K_{n}} \geq D\left|\beta_{n}\right|^{j+1}$, this is at most 1 . Since the $\tilde{q}_{n}^{\prime}$ vertical length of $\omega_{n} \in \Omega_{n}$ is less than that of $\beta_{n}$, which is 1 , and the cross product $\left|\beta_{n} \times \omega_{n}\right| \leq D$ is preserved under Teichmüller maps, (3.5) implies the horizontal length of $\omega_{n} \leq D+1$.

Thus the $\tilde{q}_{n}^{\prime}$-length of $\Omega_{n}$ is bounded. Again passing to a subsequence we may assume $Z_{n}^{\prime} \rightarrow Z_{\infty}^{\prime}$ and $\tilde{q}_{n}^{\prime} \rightarrow \tilde{q}_{\infty}^{\prime}$, where now $Z_{\infty}^{\prime}$ is a compact surface, possibly with boundary, with or without punctures.

Now we claim $\tilde{q}_{\infty}^{\prime} \neq 0$ on some component of $Z_{\infty}^{\prime}$. For if $\tilde{q}_{\infty}^{\prime} \equiv 0$ then as before there would be a subcomplex $Z_{n}^{\prime \prime}$ of $Z_{n}^{\prime}$ containing $\beta_{n}$ with boundary 
length $\left|\Omega_{n}^{\prime \prime}\right| \rightarrow 0$. Mapping back to $Z_{n}$ by the inverse Teichmüller map, we would have $\left|\Omega_{n}^{\prime \prime}\right| \leq\left|\beta_{n}\right| / 4$, a contradiction to $Z_{n}$ being the smallest surface with that property.

Thus $\tilde{q}_{\infty}^{\prime} \not \equiv 0$ and as in Case I we can find a horizontal segment of length $\geq \alpha$ disjoint from $\beta_{n}$ on $Z_{n}^{\prime}$. Mapping back to $Z_{n}$ we get a segment of $\tilde{q}_{n}$ length $\geq \delta_{n}^{1 / 2} \alpha$. Its $q_{n}$-length is at least $\delta_{n} \alpha_{n}$ and finally mapping back to $X$, the horizontal length $\geq \alpha \delta_{n} /\left|\beta_{n}\right|$. Recalling that $\delta_{n} \geq 1 / D\left|\beta_{n}\right|^{j}$ the horizontal length $\geq \alpha / D\left|\beta_{n}\right|^{j+1}$. Choose $K=j+1$ and we get a contradiction.

We now consider a horizontal trajectory $h$ of $q$ with one endpoint a zero. Recall $\theta_{\gamma}$ denotes the angle which $\gamma$ makes with the vertical direction.

Proposition 3.3. Assume the vertical trajectories of $q$ are dense. There exists $c>0$ such that for any horizontal $h$ of length $|h|$ on $X$ there is a saddle connection $\gamma$ such that

$$
|\gamma| \leq c /|h| \text { and }\left|\sin \theta_{\gamma}\right| \leq|h| /|\gamma| \text {. }
$$

If $\gamma$ is one boundary component of an annulus of closed trajectories, the closed trajectories intersect $h$. If $\gamma$ is not such a boundary component then $h_{\theta}(\gamma) \geq$ $|h| / c$.

Proof. Consider the decomposition of the surface into rectangles with horizontal sides on $h$ (cf. [S, V1]). There are at most $c_{1}$ rectangles where $c_{1}$ depends only on the genus. Each rectangle has a zero on each vertical side or at a vertex on the vertical side unless one of the sides has the nonzero endpoint of $h$ as a vertex. There is a constant $c_{2}>0$ such that the widest rectangle $R$ has width $\geq c_{2}|h|$. Its height $\leq 1 / c_{2}|h|$ since the area of any rectangle $\leq 1$.

Case I. If there are zeros on both vertical sides of $R$ join them by a saddle connection $\gamma$ across this rectangle. Then $|\gamma| \leq c /|h|$ and $\left|\sin \theta_{\gamma}\right| \leq 2|h| /|\gamma|$ for some $c$.

Case II. $R$ has a vertex at the nonzero endpoint of $h$. Call the two horizontal sides of $R, h_{R}^{1}$ and $h_{R}^{2}$ with width $\left|h_{R}\right| \geq c_{2}|h|$. If $h_{R}^{1}$ and $h_{R}^{2}$ lie on opposite sides of $h$ and overlap, we say $R$ bites on itself. This means some vertical trajectory will leave $h_{R}^{1}$, return to the opposite side of $h$ (on $h_{R}^{2}$ ), and then continue by traversing $R$ again. In this case there is a closed trajectory (not necessarily vertical) completely contained in $R$ that joins two points on the opposite horizontal sides. On the boundary of the annulus of these closed trajectories is a saddle connection $\gamma$ satisfying the conclusions.

If $R$ does not bite on itself, $h_{R}^{1}$ and $h_{R}^{2}$ are either on the same side of $h$ or if on opposite sides, they do not overlap. Find rectangles $R_{1}$ and $R_{2}$ whose horizontal sides $h_{R_{1}}$, and $h_{R_{2}}$ contain the largest part of $h_{R}^{1}$ and $h_{R}^{2}$, respectively. Their widths are $\geq c_{3}$ width $R \geq c_{3} c_{2}|h|$ for some constant $c_{3}$.

If either $R_{1}$ or $R_{2}$ has a zero on both vertical sides, again we are done by taking a saddle connection across that rectangle. If not, $R_{1}=R_{2}$ and one 
vertex of $R_{1}$ is the nonzero endpoint of $q$. There are now two cases. Assume first

$$
\left|h_{R_{1}}\right|=\text { width } R_{1} \geq 7 / 8\left|h_{R}\right| .
$$

Then there is a subinterval $h^{\prime}$ of $h_{R}^{1}$ with the property that every vertical trajectory leaving $h^{\prime}$ traverses $R$, returns to $h$ along $h_{R}^{2} \cap h_{R_{2}}$, traverses $R_{1}$, and comes back to $h_{R_{1}} \cap h_{R}^{1}$. Also some vertical trajectory leaves $h^{\prime}$ and comes back to $h^{\prime}$. That is, the interval $h^{\prime}$ bites on itself after two returns to $h$. There is then a closed trajectory across this rectangle. Its length is

$$
\leq \frac{1}{\left|h_{R}\right|}+\frac{1}{\left|h_{R_{1}}\right|}+2|h|<\frac{c}{|h|}
$$

and thus there is a saddle connection $\gamma$ of no larger length on the boundary of the annulus. The angle it makes with the vertical is $\leq 2|h| /|\gamma|$.

If $\left|h_{R_{1}}\right| \leq 7 / 8\left|h_{R}\right|$ then there is another rectangle $R_{3}$ with width $\geq c_{4}\left|h_{R}\right|$ for some $c_{4}$ overlapping $h_{R}^{1}$ and it must have zeros on both vertical sides since $R$ and $R_{1}$ are the two rectangles with the nonzero endpoint of $h$ as vertices. We now take a saddle connection $\gamma$ joining the zeros across $R_{3}$.

Proof of Proposition 2.3. For $q \notin F_{j}(\beta)$ the hypotheses of Proposition 3.2 are satisfied.

We will now choose a set of constants satisfying certain conditions. Recall the constants $j, K$, and $\alpha$ defined by Proposition 3.2 and $c$ defined by Proposition 3.3. Now choose $\alpha^{\prime}<\alpha, K^{\prime} \geq K$, and $0<r_{2}<r_{1}<1$ such that for all $x \geq 2$

(a) $j+1 \leq \min \left(K^{\prime}, 1 / r_{1}-1\right)$,

(b) $K^{\prime} r_{1} \geq 2$,

(c) $x^{2 K} \leq\left(x^{2 K^{\prime}} /(n+1)\right)\left(\alpha^{\prime} / 2 c D\right)<n x^{2 K^{\prime}}$, and

(d) $8 c x^{3 r_{2}+3}(x+1)^{2} \leq x^{2 K^{\prime}\left(r_{1}-r_{2}\right)}$.

Now let $L=\left(\alpha^{\prime} / 2 c D\right)\left(|\beta|^{K^{\prime}} /(n+1)\right)$. Since $|\beta| \geq n^{2} \geq 4,|\beta|^{K} \leq L \leq n|\beta|^{K^{\prime}}$ by (c). Then by Proposition 3.2, for any $k \geq K^{\prime}$ and $\theta$ such that

$$
\left|\sin \frac{\theta}{2}\right| \leq \frac{1}{n|\beta||\beta|^{k}}\left(1-\left(\frac{1}{n|\beta||\beta|^{k}}\right)^{r_{2}}\right) \leq \frac{1}{n|\beta||\beta|^{k}} \leq \frac{1}{|\beta| L},
$$

there is a horizontal trajectory disjoint from $\beta$ with one endpoint a zero and length at least

$$
\frac{\alpha^{\prime}}{D L}=\frac{2 c(n+1)^{2}}{|\beta|^{K^{\prime}}} \geq \frac{2 c(n+1)^{2}}{|\beta|^{k}} .
$$

Take a horizontal trajectory $h$ with length $|h|=2 c(n+1)^{2} /|\beta|^{k}$. By Proposition 3.3 there is a saddle connection $\gamma$ such that

$$
|\gamma| \leq c /|h| \leq|\beta|^{k} / 2(n+1)^{2}
$$


and

$$
\left|\sin \frac{\left(\theta-\theta_{\gamma}\right)}{2}\right| \leq \frac{2|h|}{|\gamma|} \leq \frac{4 c(n+1)^{2}}{|\gamma||\beta|^{k}} .
$$

It is clear $\theta_{\gamma} \in I$ if $|\beta|$ is sufficiently large so $q \notin F_{j}(\gamma)$. We first need to check $\gamma \neq \beta$. If $\gamma$ joins two zeros across a rectangle then

$$
h_{\theta}(\gamma) \geq|h| / c \geq 2(n+1)^{2} /|\beta|^{k} .
$$

However (3.6) implies $h_{\theta}(\beta) \leq 1 / n|\beta|^{k}$ so $\gamma \neq \beta$. If on the other hand $\gamma$ was constructed from an annulus of closed trajectories, $\gamma$ crosses $h$ and since $\beta$ does not, again $\gamma \neq \beta$.

Now by (3.6), since $\beta$ is vertical

$$
|\beta \times \gamma|=|\beta| h_{\theta}(\gamma)=|\beta|\left|\sin \frac{\theta}{2}\right||\gamma| \leq \frac{|\gamma|}{n|\beta|^{k}} .
$$

If $|\gamma| \leq|\beta|$ then (3.9) and (a) give

$$
|\beta \times \gamma| \leq \frac{1}{n|\beta|^{k-1}} \leq \frac{1}{n|\beta|^{K^{\prime}-1}} \leq \frac{1}{|\beta|^{j}},
$$

contradicting $q \notin F_{j}(\beta)$.

If $|\beta| \leq|\gamma| \leq|\beta|^{k r_{1}}$ then (3.9) and (a) give

$$
|\beta \times \gamma| \leq|\gamma|^{1-1 / r_{1}} / n \leq 1 /|\gamma|^{j}
$$

and this contradicts $q \notin F_{j}(\gamma)$. Thus by (b)

$$
|\gamma| \geq|\beta|^{k r_{1}} \geq|\beta|^{2}
$$

so (ii) holds. We now check the rest of the conclusions of the proposition. Now (3.8), (3.10), (d), and the fact that $|\beta| \geq n^{2}$ give

$$
\begin{aligned}
\left|\sin \frac{\left(\theta-\theta_{\gamma}\right)}{2}\right| & \leq \frac{4 c(n+1)^{2}}{|\gamma||\beta|^{k}} \leq \frac{4 c(n+1)^{2}}{|\beta|^{k}|\beta|^{k r_{1}}}=\frac{4 c(n+1)^{2} n}{n|\beta||\beta|^{k}|\beta|^{k r_{1}-1}} \\
& \leq \frac{1}{2}\left(\frac{1}{n|\beta||\beta|^{k}}\right)^{1+r_{2}} \leq|I(\beta, n, 2 k \log |\beta|)|^{1+r_{2}} .
\end{aligned}
$$

Thus (iv) holds. Also by (3.6) and (3.11)

$$
\left|\sin \frac{\theta_{\gamma}}{2}\right| \leq\left|\sin \frac{\left(\theta-\theta_{\gamma}\right)}{2}\right|+\left|\sin \frac{\theta}{2}\right| \leq \frac{1}{n|\beta||\beta|^{k}},
$$

so (i) holds as well. Finally (3.7) and (3.12) give

$$
|\gamma| \sin \frac{\theta_{\gamma}}{2} \mid \leq \frac{|\beta|^{k}}{2(n+1)^{2} n} \frac{1}{|\beta||\beta|^{k}}=\frac{1}{2 n(n+1)^{2}|\beta|},
$$

so (iii) follows as well. 


\section{Measure classes on $Q_{k}^{0} /$ Map}

Given $q_{0} \in Q_{k}$ and a finite set $\gamma$ of saddle connections of $q_{0}$, orient each $\gamma$ and choose a branch of $q_{0}^{1 / 2}$ along each. Then for $q$ in a neighborhood $U \subset Q_{k}$ of $q_{0}$, with codimension 1 boundary, each oriented $\gamma$ is a saddle connection of $q$ and we can continuously pick a branch of $q^{1 / 2}$.

There is a finite set $\gamma_{1}, \ldots, \gamma_{r}$ such that the map $F: U \rightarrow \mathbf{R}^{2 r}$ given by

$$
F(q)=\left(\int_{\gamma_{1}} \operatorname{Re} q^{1 / 2} d z, \int_{\gamma_{1}} \operatorname{Im} q^{1 / 2} d z, \ldots, \int_{\gamma_{r}} \operatorname{Im} q^{1 / 2} d z\right)
$$

is a local homeomorphism.

If $q$ is the square of an abelian differential, $\gamma_{1}, \ldots, \gamma_{r}$ is a basis for $H_{1}(M, \Sigma, \mathbf{R})$ where $\Sigma$ is the set of zeros of $q$. If $q$ is not a square, form the orientable double cover defined by $q^{1 / 2}$, ramified over the odd zeros of $q$. Then form the singular surface by identifying pairs of points that lie over the same even order zero. This singular surface carries a natural involution which interchanges the sheets of the cover. Then $\gamma_{1}, \ldots, \gamma_{r}$ is a set of saddle connections whose lifts to this double cover form a basis for the homology which is odd with respect to the involution.

Define a measure $\lambda$ on $U$ as the measure induced from product Lebesgue measure on $\mathbf{R}^{2 r}$ by $F$. The boundary has measure zero. Covering $Q_{k}$ disjointly by such sets gives a measure $\lambda$. We need to define a measure $\tilde{\lambda}$ on $Q_{k}^{0}$. For $E \subset Q_{k}^{0}$ let

$$
\tilde{\lambda}(E)=\lambda(\{t q: q \in E: 0 \leq t \leq 1\}) .
$$

Now $\tilde{\lambda}$ is invariant under Map and under the flow $g_{t}$.

One set of $\gamma_{i}$ that we can use are saddle connections that are edges of a canonical triangulation of the surface defined by the quadratic differential. This triangulation was found in [MS]. We give its main properties. The shortest saddle connection (or connections) is an edge (or edges) of the triangulation and the next shortest edge has length at most a fixed constant times the length of the next shortest saddle connection. Each edge has length at most the diameter of the surface. Moreover there are a finite number of triangulations such that each quadratic differential in the quotient space has one of these triangulations as its canonical triangulation. This means that we only need to make estimates for the quadratic differentials that have a given triangulation as their canonical triangulation.

For the rest of this section $C$ with a subscript will refer to any universal constant. Choose a simply connected set of full measure in $Q_{k}^{0} /$ Map without singularities so it is possible to consistently speak of $\beta$ as a saddle connection for all quadratic differentials in the set.

For any saddle connection $\beta$ let $E(\beta, j, L, \varepsilon, M)$ be the set of $q:|\beta|_{q} \leq \varepsilon$ and either 
(i) $\beta$ is part of or equal to one boundary component of an annulus of closed trajectories and the complement has area $\leq 1 / L^{j}$, or

(ii) there is a subsurface with area $\leq 1 / L^{j}$ containing $\beta$ in its interior whose boundary consists of saddle connections $\gamma$ such that $|\gamma| \leq M|\beta|$.

Lemma 4.1. There are constants $C_{1}$ and $L_{0}$ such that for each $j$ and $L \geq L_{0}$

$$
\tilde{\lambda}(E(\beta, j, L, \varepsilon, M)) \leq C_{1}\left(M \varepsilon / L^{j}+\varepsilon^{4}\right) .
$$

Proof. We begin by noting the following simple computation. The Lebesgue measure in $\mathbf{R}^{4}$ of $\left\{\left(x_{1}, y_{1}, x_{2}, y_{2}\right):\left|\left(x_{1}, y_{1}\right)\right| \leq \varepsilon,\left|\left(x_{2}, y_{2}\right)\right| \leq M^{\prime}\right.$, and $\left.\left|y_{2} x_{1}-x_{2} y_{1}\right| \leq 1 / L^{j}\right\} \leq C_{2} M \varepsilon / L^{j}$ for some $C_{2}$.

Next by Lemma 4.3 of [M2] there is a constant $C_{3}$ such that if the diameter of the surface defined by the metric $\left|q^{1 / 2}\right| \geq C_{3}$, there is an annulus of closed trajectories whose width is $\geq C_{3}$. Moreover, the circumference and saddles crossing the annulus are edges. Consider any such annulus with area $\leq 1$ and with length at most $\varepsilon$. The shortest saddle connection crossing the annulus has length $\leq \varepsilon+1 / \varepsilon$. The coordinates $(x, y)$ of this curve define a subset of $\mathbf{R}^{2}$. It follows that the Lebesgue measure of this subset is uniformly bounded independent of $\varepsilon$. To see that, we may assume without loss of generality that the vertical coordinate of the annulus is larger than the horizontal coordinate. Then for fixed $x$ the value of $y$ lies in an interval of width at most $c \varepsilon$ for $c$ a constant. Since $x \leq \varepsilon+1 / \varepsilon$, the estimate follows by Fubini's Theorem.

This computation says that in the computation of the product Lebesgue measure in $\mathbf{R}^{2 r}$ defined by the coordinates of the edges of a canonical triangulation, the coordinates of long edges that come from metrics with large diameter define sets of bounded measure.

Consider now a canonical triangulation. Consider the set of quadratic differentials for which $\beta$ is not an edge. Then there is a shorter saddle connection $\alpha$ which is an edge. If $\alpha$ is not homologous to $\beta$ there is an edge $\gamma$ not homologous to $\alpha$ of length at most $C \varepsilon$ for some constant $C$. We have two nonhomologous edges each with coordinates at most $C \varepsilon$. The measure in $\mathbf{R}^{4}$ defined by these coordinates is $\leq C^{4} \varepsilon^{4}$. The above discussion on the diameter shows that the contribution to the product Lebesgue measure defined by other edges is uniformly bounded and so the product measure defined by all edges is the second term in the sum. If $\alpha$ is homologous to $\beta$, then since $\beta$ is not an edge, there is an edge $\gamma$ as above.

Thus we may consider the set of quadratic differentials such that $\beta$ is an edge. If (i) holds then we may assume that $\beta$ is not on the boundary of another annulus of closed trajectories, for the set of quadratic differentials with the property that a saddle connection is on the boundary of two annuli has measure zero. We can also assume the diameter of the complement $\leq C_{3}$ by removing other annuli. Assumption (i) allows us to find an edge $\gamma$ of length $\leq C_{3}$ which together with $\beta$ bound two sides of a geodesic triangle of area $\leq 1 / L^{j}$. This 
implies that $|\gamma \times \beta| \leq 2 / L^{j}$. The estimate on measure in $\mathbf{R}^{4}$ given above and Fubini's theorem give the required estimate for this set. Again note that the contribution of other coordinates to the product measure is bounded.

If (ii) holds and the boundary curve is not homologous in the double cover to $\beta$, we can find a second edge not homologous to $\beta$ with length $\leq C \varepsilon$ and we are in a case previously considered. If the boundary is homologous, then if it is not in the triangulation there is an edge of length $\leq C \varepsilon$ which is and we are again in a case previously considered. If the boundary is in the triangulation, the area assumption on the subsurface allows us to find a $\gamma$ as before.

Proposition 4.2. For $j$ sufficiently large, $\tilde{\lambda}\left(F_{j}\right)=0$.

Proof. For any compact subset $K$ of $Q_{k}^{0} /$ Map there is a $c_{1}=c_{1}(K)$ such that the ratio of lengths of any geodesic joining singularities measured in any two metrics of $K$ is bounded. Fix $L_{0}$ and $\rho>1$. There are constants $c_{2}=c_{2}(q)$ and $p$ such that the number of geodesics of $q$ of length $<\rho^{l} L_{0}$ is at most $c_{2} L_{0}^{p} \rho_{0}^{l p}$. Here $p$, the growth rate, does not depend on $q$, only on the genus. Fix $q_{0}$ and $c_{2}=c_{2}\left(q_{0}\right)$.

Divide $[0,2 \pi]$ into disjoint intervals $I_{i}$ of equal width, each approximately

$$
\frac{1}{2}\left(\rho^{l+1} L_{0}\right)^{-j-2} .
$$

Let $I_{1}$ be the one with endpoint 0 and for each $i$ let $\theta_{i}$ be such that $I_{i}-$ $\theta_{i}=I_{1}$. For each saddle connection $\beta$ let $K_{i}(\beta, l)=\{q \in K: \beta$ is a saddle connection of $q$ of length between $\rho^{l} L_{0}$ and $\rho^{l+1} L_{0}$ and $\left.\theta_{\beta} \in I_{i}\right\}$.

Let $e^{t / 2}=\sqrt{2}\left(\rho^{l+1} L_{0}\right)^{j / 2+1}$. For $q \in K_{i}(\beta, l) \cap F_{j}(\beta)$, consider $g_{t}\left(e^{i \theta_{i}} q\right)$. Since $\left|\beta_{q}\right| \leq \rho^{l+1} L_{0}$ and $\theta_{\beta} \in I_{i}$ we have

$$
h_{\theta_{i}}(\beta)=|\beta|\left|\sin \frac{\left(\theta_{i}-\theta_{\beta}\right)}{2}\right| \leq \frac{1}{2} \frac{1}{\left(\rho^{l+1} L_{0}\right)^{j+1}} .
$$

Thus both $e^{t / 2} h_{\theta_{i}}(\beta)$ and $e^{-t / 2} v_{\theta_{i, t}}(\beta) \leq\left(\rho^{l+1} L_{0}\right)^{-j / 2} / \sqrt{2}$. Thus $|\beta|_{\theta_{i, t}} \leq$ $\varepsilon=\left(\rho^{l+1} L_{0}\right)^{-j / 2}$. A similar computation shows that if $|\gamma \times \beta| \leq 1 /|\beta|^{j}$ and $|\gamma| \leq|\beta|$, then $|\gamma|_{\theta_{i, t}} \leq \sqrt{2} \rho^{j+1} \varepsilon$.

Now the Teichmüller map preserves cross products and area elements, so $g_{t}(q) \in E\left(\beta, j, \rho^{l} L_{0}, \varepsilon, \sqrt{2} \rho^{j+1}\right)$. For fixed $t, g_{t}$ preserves $\tilde{\lambda}$. Thus by Lemma 4.1, for some universal $c$,

$$
\tilde{\lambda}\left(K_{i}(\beta) \cap F_{j}(\beta)\right) \leq c\left(\frac{1}{\left(\rho^{l+1} L_{0}\right)^{2 j}}+\frac{\rho^{j+1} \rho^{j}}{\left(\rho^{l+1} L_{0}\right)^{3 j / 2}}\right) .
$$

The second term dominates. Since there are $4 \pi\left(\rho^{l+1} L_{0}\right)^{j+2}$ such indices $i$,

$$
\tilde{\lambda}\left(K \cap F_{j}(\beta)\right) \leq \frac{c \rho^{2 j+1}}{\left(\rho^{l+1} L_{0}\right)^{2+j / 2}}
$$

for some new $c$. 
If $\beta$ has length at most $\rho^{l+1} L_{0}$ on $q$, it has length at most $c_{1} \rho^{l+1} L_{0}$ on $q_{0}$. There are at most $c_{2} c_{1}^{p} L_{0}^{p} \rho^{(l+1) p}$ such $\beta$. Summing over all $l$ we have

$$
\sum_{\beta} \tilde{\lambda}\left(K \cap F_{j}(\beta)\right) \leq \sum_{l=1}^{\infty} \frac{c c_{1}^{p} c_{2} L_{0}^{p} \rho^{(l+1) p} \rho^{2 j+1}}{\left(\rho^{l+1} L_{0}\right)^{2+j / 2}} .
$$

Now choose $j$ sufficiently large so this series converges. $j$ does not depend on the compact set $K$. Thus for any compact $K$ we have by the Borel-Cantelli Lemma

$$
\tilde{\lambda}\left(K \cap F_{j}\right)=0 \text {. }
$$

Proof of Theorem 2. Fix $j$ so Proposition 4.2 holds. Proposition 2.4 then says for $\tilde{\lambda}$ a.e. $q \in Q_{0}^{k}$ there is a $\delta=\delta(q)>0$ such that the Cantor set of divergent geodesics has Hausdorff dimension $\delta$. The Hausdorff dimension $\delta$ is a measurable function of $q$ and invariant under the flow $g_{t}$. To see this, notice any cover $I_{i}$ of the divergent set of angles defined by $q$ induces a cover $I_{i}^{\prime}$ of the set defined by $g_{t}(q)$ where $a\left|I_{i}\right| \leq\left|I_{i}^{\prime}\right| \leq b\left|I_{i}\right|$ for some $a, b$ depending only on $t$.

Now the conclusion, $\delta_{0}$ constant, follows from the fact that the flow $g_{t}$ is ergodic on $Q_{0}^{k}$ [V2].

Proof of Theorem 1 . We can locally write $Q_{k}^{0}$ as $[0,2 \pi) \times I$ for an interval $I$ of dimension $n-1=\operatorname{dim} Q_{k}^{0}-1$ and such that for $y \in I,\{(\theta, y): \theta \in[0,2 \pi)\}=$ $\left\{e^{i \theta} q: \theta \in[0,2 \pi)\right.$ for some $\left.q\right\}$. Theorem 2 says for a.e. $y \in I$, the set $C(y)=\{\theta:(\theta, y)$ is divergent $\}$ has Hausdorff dimension $\delta$. Then for $\delta_{1}<\delta$, $H^{\delta_{1}}(C(y))=\infty$ for a.e. $y$. Theorem 5.8 of $[\mathrm{F}]$ says $H^{n-1+\delta_{1}}([0,2 \pi) \times I)=\infty$. Theorem 1 follows.

\section{REFERENCES}

[B] A. Beardon, The Hausdorff dimension of singular sets of properly discontinuous groups, Amer. J. Math. 88 (1966), 721-736.

[Ber] L. Bers, Finite dimensional Teichmüller spaces and generalizations, Bull. Amer. Math. Soc. 5 (1981), 131-172.

[F] K. J. Falconer, The geometry of fractal sets, Cambridge Univ. Press, Cambridge, 1985.

[Fa] A. Fathi, et. al, Travaux de Thurston sur les surfaces, Asterisque 66-67 (1979).

[G] F. Gardiner, Teichmüller theory and quadratic differentials, Wiley, New York, 1987.

[KMS] S. Kerckhoff, H. Masur, and J. Smillie, Ergodicity of billiard flows and quadratic differentials, Ann. of Math. (2) 124 (1986), 293-311.

[K] S. Kerckhoff (to appear).

[M1] H. Masur, Closed trajectories for quadratic differentials with an application to billiards, Duke Math. J. 53 (1986), 307-314.

[M2] _ Interval exchange transformations and measured foliations, Ann. of Math. (2) 115 (1982), 169-200.

[M3] _ The asymptotics of sadale connections for a quadratic differential, Ergodic Theory Dynamical Systems 10 (1990), 151-176. 
[MS] H. Masur and J. Smillie, Hausdorff dimension of sets of nonergodic measured foliations, preprint.

[R] M. Rees, An alternative approach to the ergodic theory of measured foliations on surfaces, Ergodic Theory Dynamical Systems 1 (1981), 461-485.

[S] K. Strebel, Quadratic differentials, Springer-Verlag, Berlin and New York, 1984.

[V1] W. Veech, Gauss measures for transformations on the space of interval exchange maps, Ann. of Math. (2) 115 (1982), 201-242.

[V2] _ The Teichmüller geodesic flow, Ann. of Math. (2) 124 (1986), 441-530.

[V3] _ Teichm̈uller curves in moduli space, Einstein Series and an Application to Triangular Billiards, Invent. Math. 97 (1989), 553-583.

Department of Mathematics, Statistics, and COMPuter SCIENCE, UNiversity of Illinois at Chicago, Chicago, Illinois 60680 